\title{
Theorien der Stadt
}

Rezension zu Lars Meier / Silke Steets / Lars Frers (2018): Theoretische Positionen der Stadtsoziologie. Weinheim: Beltz Juventa.

Jan Wehrheim

Abb. 1 Titel des Buches (Quelle: Beltz Juventa Verlag)

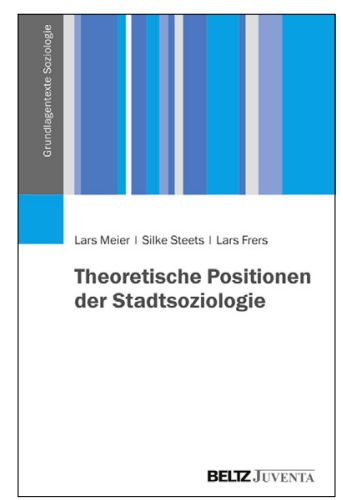

Mitte der 200oer Jahre gab es letztmalig eine kleine Welle an deutschsprachigen Einführungs- und Lehrbüchern zur Stadtsoziologie. Gut zehn Jahre später ist dies erneut der Fall. Während zwei andere deutschsprachige Neuerscheinungen explizit auf das Verhältnis von Stadtsoziologie und Sozialer Arbeit zielen respektive die Relevanz der ersteren Disziplin für die letztere hervorheben (Baum 2018; Herrmann 2018), richtet sich der hier besprochene Band an alle an Stadt Interessierten und damit auch gleichermaBen an Lernende wie Lehrende. An Erstere, weil das Buch in unterschiedliche theoretische Positionen und das Werk damit verknüpfter Autor*innen einführt. An Letztere richtet es sich, weil es sich durchaus zur Vorbereitung der Lehre eignet und dafür, einführende Texte für Studierende bereitzustellen, aber natürlich auch, weil auch die Lehrenden meist nicht alle Zugänge zur Stadtsoziologie detailliert kennen oder einzelne gern einmal auffrischen.

In diesem Sinne ist das Buch auch strukturiert und aufgebaut. Die drei Autor*innen - das Buch ist kein Sammelband, sondern die Kapitel sind offenbar gemeinsam verfasst worden - entscheiden sich zunächst für vier thematische Zugänge beziehungsweise empirische Gegenstände: Transformationen, Wachstum und Schrumpfung, Zentrum und Peripherie sowie soziale Ungleichheit. Diese thematische Strukturierung wird ergänzt durch glossarartige Kästen zu zentralen Begriffen oder Konzepten: von ,Dialektik über ,Gender bei Georg Simmel' und ,Los Angeles School of Urbanism‘ bis zu ,Segregation‘, ,Taylorismus‘ und ,Weltsystemtheorie von Immanuel Wallerstein'. Diese Exkurse und Begriffserklärungen deuten schon an, dass es, um Stadt als soziales Gefüge zu verstehen, nicht reicht, nur zentrale stadtsoziologische Begrifflichkeiten zu kennen. Eine Auseinandersetzung mit anderen Grundbegriffen der nicht stadtspezifischen Soziologie, wie zum Beispiel Kolonialismus, Neoliberalismus, Alltag oder Habitus, ist zusätzlich essentiell. Die genannten vier thematischen Blöcke sind wiederum in zwei bis vier Unterkapitel gegliedert, wobei diese sich an einschlägigen, überwiegend in der Soziologie beheimateten Stadtforscher*innen orientieren und nicht etwa an Theorien wie einem materialistischen oder system- oder dependenztheoretischen Zugriff auf Stadt. Zu nennen sind in der 
Reihenfolge, wie sie im Buch auftauchen: Georg Simmel, David Harvey, Sharon Zukin, Richard Sennett, Robert E. Park, Mike Davis, Henri Lefebvre, Saskia Sassen, Jennifer Robinson, Norbert Elias und Loïc Wacquant. Die ersten gut 100 Jahre expliziter Stadtforschung beziehungsweise ganz überwiegend soziologischer Stadtforschung sind also - zwangsläufig unvollständig - von den Autor*innen aufgegriffen worden. Gegliedert sind die den Oberthemen zugeordneten Kapitel zu den Theoretiker*innen immer nach demselben Muster: Einführung zum*r Autor*in, Theorieposition, Kritik und Weiterentwicklung sowie Zusammenfassung. Lernfragen (die auch den Eindruck von möglichen Klausurfragen machen) ergänzen die Kapitel.

Das Buch ist mit diesem Aufbau und seinem Inhalt als durchaus gelungen anzusehen. Ein entsprechendes Buch existierte im deutschsprachigen Raum bislang nicht und Einführungen in das Werk einzelner Autor*innen erfolgten in anderen Lehr- und Einführungsbüchern eher nebenbei (Peter Saunders Soziologie der Stadt von 1987 war eine Ausnahme) oder es wird im dicken Handbuchformat am Beispiel von ,Klassikern' auf soziologische Hintergründe/Referenzpunkte für die Stadtforschung eingegangen (vgl. Eckardt 2012). Wer eine gut verständliche und systematische Einführung in unterschiedliche theoretische Perspektiven auf Stadt lesen oder schnell verstehen möchte, warum es immer noch gewinnbringend ist, beispielsweise in Bezug auf Machtfragen und soziale Beziehungen Norbert Elias zu lesen oder wieso aktuell Entwicklungen in deutschen oder südafrikanischen Großstädten besser erklärt werden können, wenn man auch die Werke von Zukin aus den 1980er und 9oer Jahren zu Zusammenhängen von Kultur und Gentrification gelesen hat, dem hilft das Buch weiter. Was das Buch im Unterschied zu anderen Lehr- oder Einführungsbüchern zur Stadtsoziologie logischerweise nicht dezidiert bietet, ist eine systematische Einführung in typische Gegenstände der Stadtforschung: Die Produktion von Stadt, residentielle Segregation, Wandel des öffentlichen Raums et cetera.

Mit Blick auf die vier Themenbereiche und auf das Werk der elf herausgehobenen Stadtforscher*innen wird allerdings auch schnell deutlich, dass es die optimale Gliederung für so ein Buch nicht geben kann. Die Auswahl erscheint nachvollziehbar, aber es hätten ebenso auch andere Theoretiker*innen gewählt werden können - von Friedrich Engels und Karl Marx über Hans Paul Bahrdt und Manuel Castells bis zu Sudhir Venkatesh. Eine Auswahl muss gleichwohl getroffen werden und die genannten Autoren tauchen durchaus auch in den Kapiteln zu passenden anderen Autor*innen, etwa im Harvey-Kapitel, auf - aber nur nebenbei. Dass der Eigenlogikansatz von Helmut Berking und Martina Löw zwar erwähnt wird, aber von deren drei ehemaligen Doktorand*innen, die auch selbst mit ihm gearbeitet haben, nicht als eigenständiger Ansatz aufgenommen wurde, überrascht jedoch.

Das Buch über Namen und Theoriepositionen zu gliedern, führt aber auch dazu, dass die Verbindung nicht immer stringent durchgehalten wird. Bei Davis wird eher auf die ganze sogenannte Los Angeles School eingegangen, während Sennetts Werk in der Breite dargestellt wird und so kaum von einem theoretischen Zugang oder einem Empirieprogramm mit Fokus auf Stadt gesprochen werden kann. Wobei sich gerade in Bezug auf Sennett der Eindruck aufdrängt, dass es bei aller Prominenz eben auch nicht schlimm ist, wenn man bisher nicht alle Theoretiker*innen der Stadt detailliert zur 
Kenntnis genommen hat. Insofern wird in dem Buch auch deutlich, wie sehr sich die Theoriepositionen respektive die Publikationen der referierten Autor*innen in ihrer Klarheit und Stringenz, aber auch in ihrem Gehalt und ihrer Verständlichkeit unterscheiden.

$\mathrm{Zu}$ Recht schreiben Meier, Steets und Frers, dass die Kapitel unabhängig voneinander gelesen werden können, das Buch also als eine Art Nachschlagwerk genutzt werden kann. Trotzdem ist die Gesamtlektüre zu empfehlen, denn nicht alle relevanten Aspekte werden für alle Autor*innen entsprechend differenziert dargelegt. Vermisst man etwa bei Harvey zunächst einen näheren Eindruck, warum von einer politischen Ökonomie gesprochen werden muss, so wird dies später bei Zukin umso anschaulicher. Nicht ganz klar ist manchmal auch, warum bestimmte Kritikpunkte an einzelnen Ansätzen aufgegriffen werden, andere jedoch nicht: Die marxistisch informierte Kritik an der Chicago School und Park fehlt etwa, die Kritik an Wacquant, er stelle Menschen in Armut zu sehr als passive Opfer des Neoliberalismus dar, wird hingegen angeführt. Umso überzeugender sind wiederum die Stellen, an denen Querbezüge zwischen den Ansätzen hergestellt werden. So werden etwa zu Robinsons Überlegungen zur postkolonialen Stadt die Bezüge zu Simmel, Park und Sassen herausgearbeitet. Anschaulich sind ebenfalls Beispiele, anhand derer theoretische Positionen verdeutlicht werden, sowie, leider seltene, Verweise auf methodologische Fragen. Die Frage, welches Wissen über Stadt in welchen Verbindungen von Theoriepositionen und Forschungsmethoden hervorgebracht wird, würde aber wohl auch ein eigenes Buch verdienen.

Insgesamt überzeugt das Buch; die kleinen Schwächen erscheinen geradezu seinem Gegenstand immanent zu sein, und es zeigt, wie intensiv sich die drei Autor*innen mit den Werken (und der dazugehörigen Sekundärliteratur) auseinandergesetzt haben. Vieles davon wurde, so ist der Danksagung zu entnehmen, bereits in der Lehre erprobt - vermutlich mit Erfolg.

Dieser Artikel wurde durch den Open-Access-Publikationsfonds der Universität Duisburg-Essen gefördert.

\section{Autor innen}

Jan Wehrheim ist Sozialwissenschaftler und Entwicklungspolitologe, forscht und lehrt seit vielen Jahren in Feldern der Stadt-, Devianz- und Techniksoziologie.

jan.wehrheim@uni-due.de

\section{Literatur}

Baum, Detlef (2018): Lehrbuch Stadt und Soziale Arbeit. Stadtsoziologische Grundlagen Sozialer Arbeit. Weinheim: Beltz/Juventa.

Eckardt, Frank (Hg.) (2012): Handbuch Stadtsoziologie. Wiesbaden: Springer.

Herrmann, Heike (2018): Soziale Arbeit im Sozialraum. Stadtsoziologische Zugänge. Stuttgart: Kohlhammer.

Meier, Lars / Steets, Silke / Frers, Lars (2018): Theoretische Positionen der Stadtsoziologie. Weinheim: Beltz Juventa.

Saunders, Peter (1987): Soziologie der Stadt. Frankfurt am Main/New York: Campus. 